\title{
11 E-Assessment Service Unit: Beratung, Konzeption und Durchführung von E-Klausuren
}

\author{
Anja Richter, Manfred Daniel
}

\subsection{Die Entwicklung von E-Klausuren im Projekt optes}

Seit Beginn des optes-Projekts im Jahr 2012 werden an der Dualen Hochschule Baden-Württemberg (DHBW) Karlsruhe die technischen und organisatorischen Voraussetzungen für die Durchführung elektronischer Klausuren erforscht und in Lehrveranstaltungen umgesetzt.

In der ersten Förderphase stand vor allem die Erprobung und Anwendung der technischen Voraussetzungen und organisatorischer Abläufe rechtssicherer E-Klausuren im Vordergrund. So wurden beispielsweise verschiedene elektronische Signaturverfahren getestet und Softwareentwicklungen im Bereich Test und Assessment der Lernplattform ILIAS unterstützt. Für die Durchführung der EKlausuren wurde ein E-Klausur-Prozess definiert und im Studiengang Wirtschaftsinformatik der DHBW Karlsruhe in der Lehrveranstaltung Mathematik pilotiert.

In der zweiten Förderphase im Jahr 2014 wurde eine E-Assessment Service Unit (EASU) konzipiert und zum Einsatz gebracht, die Lehrende an der DHBW Karlsruhe bei der Erstellung und Durchführung von E-Klausuren unterstützt und damit die Anwendung des E-Klausur-Prozesses auf weitere Fachbereiche ausweitete.

Kennzeichnend für die Herausforderungen bei der Einführung von E-Klausuren sind dabei die typischen Rahmenbedingen an kleineren Hochschuleinrichtungen. An der DHBW Karlsruhe studieren im Frühjahr 20203243 Studierende in kleinen Gruppen von 25 bis 35 Studierenden pro Kurs. Ein Studiengang setzt sich in der Regel aus mehreren Kursen, teilweise mit unterschiedlichen Studienschwerpunkten, zusammen.

Die E-Klausuren können derzeit in drei PC-Räumen durchgeführt werden, die mit geeigneten gleichwertigen Arbeitsplätzen für insgesamt 30 bis 40 Studierende pro Raum ausgestattet sind. Da in Klausuren nach Möglichkeit ein freier Platz zwischen zwei Studierenden eingerichtet werden sollte, liegt die maximale Anzahl von Studierenden, die gleichzeitig an einer E-Klausur teilnehmen können, ungefähr bei 70. Bei Studierendenzahlen von circa 140, wie beispielsweise EKlausuren mit fünf Kursen der Wirtschaftsinformatik, hat sich eine zweizügige Durchführung der E-Klausur bewährt. Dabei wird ein erster Klausurdurchlauf mit 
70 Studierenden und unmittelbar im Anschluss ein zweiter Durchlauf mit einer etwas modifizierten Klausur angeboten. An der DHBW Karlsruhe lehren rund 700 Lehrbeauftragte und rund 70 hauptamtliche Professor*innen, die je nach Studienfach und zeitlicher Verfügbarkeit unterschiedliche Erwartungen und Voraussetzungen bei der Beratung, Konzeption und Durchführung von E-Klausuren mitbringen.

\subsection{Ziele und Aufgaben der E-Assessment Service Unit (EASU)}

E-Assessment in der Hochschullehre ermöglicht interaktives, mediengestütztes Testen in Lehrveranstaltungen oder als E-Klausur. Neue mediale Elemente wie Video, Audio, interaktive Abbildungen, etc. in Fragestellungen erlauben den Einsatz in verschiedenen Fachrichtungen. Außerdem erhalten die Studierenden bei Bedarf differenzierte elektronische Rückmeldungen und Lernhinweise zu ihren Antworten. Digitale Fragen können einfacher zwischen Dozierenden ausgetauscht und gemeinsam bearbeitet werden.

Zur Unterstützung der Einführung von E-Assessment-Angeboten in Lehrveranstaltungen wurde mit der EASU ein neues Beratungsangebot des Education Support Centers (ESC) an der DHBW Karlsruhe etabliert. Der Service richtet sich an Lehrende und umfasst formatives E-Assessment sowie summatives EAssessment in Form von elektronischen Prüfungen.

Die EASU berät und unterstützt Lehrende bei den folgenden Anliegen:

- Beratung zum Einsatz von formativen und summativen Assessments im Studienmodell der DHBW

- Unterstützung beim Aufbau und der Strukturierung von Fragenpools in Lehrveranstaltungen des MINT-Bereichs und in weiteren Fachrichtungen

- Beratung zu Einsatz und Umsetzung von E-Klausuren

- Unterstützung bei der Durchführung von E-Klausuren

- Beratung zur didaktischen und technischen Integration von E-Assessment in Lehrveranstaltungsabläufen

- Anleitung zur Eigenproduktion von E-Tests und E-Klausuren

- Erprobung neuer elektronischer Prüfungsformate

- Softwareentwicklungen und Verbesserungsvorschläge im E-Assessment-Bereich der Lernplattformen ILIAS und Moodle

Die Pilotierung der EASU ist Bestandteil der Projektziele des optes-Teilprojekts „E-Assessment im Studium“. Die Beratungs- und Supportaufgaben werden in diesem Rahmen von zwei Projektmitarbeiter*innen ausgeführt, die sich in zwei Arbeitsbereichen organisiert haben: 
1) der Bereich der Beratung und Umsetzung sowie der Organisation und anschließenden Evaluation von E-Assessment in Lehrveranstaltungen,

2) der Bereich der technischen Weiterentwicklung, des Software Testing sowie der Administration der Basisinfrastruktur und der Lernplattform für die technische Ausführung von E-Assessment.

Bei der Beratung der Lehrenden wird prinzipiell von den didaktischen Anforderungen der einzelnen Lehrveranstaltungen ausgegangen und danach bestimmt, mit welchen technischen Lösungen diese am besten umgesetzt werden können.

\subsection{Der E-Klausur-Prozess an der DHBW Karlsruhe und die Beratung dazu}

Im Folgenden wird der E-Klausur-Prozess vorgestellt, wie er an der DHBW Karlsruhe zurzeit stattfindet. Dabei wird wiederholt darauf eingegangen, welcher Beratungs- und Unterstützungsbedarf bei Lehrenden besteht und wie der EASU-Service hierzu aussieht. Die besonderen technischen Aspekte des E-Klausur-Prozesses werden in Kapitel 12 behandelt. Dieser Prozess ist das Ergebnis mehrjähriger Erfahrungen mit der Erstellung und Durchführung von E-Klausuren im Projekt optes. Er wurde mehrfach überarbeitet und im Detail präzisiert. Der E-KlausurProzess gliedert sich im Wesentlichen in die drei Hauptphasen Vorbereitung, Durchführung und Nachbereitung, die durch das Fachpersonal der EASU unterstützt und ausgeführt werden.

\subsubsection{Die Vorbereitungsphase}

Interessieren sich Lehrende zum ersten Mal für die Umsetzung einer E-Klausur, kann die Vorbereitungsphase bis zu einem Jahr vor dem eigentlichen Prüfungstermin beginnen, und sie endet ungefähr vier Wochen vor diesem Datum.

Wurden bereits Klausuren geschrieben und die Lehrenden kennen sich mit der Erstellung einer E-Klausur bereits aus, beginnt diese Phase in der Regel, sobald der Studienplan und damit auch der Prüfungszeitraum für die die Lehrveranstaltung feststeht. In dieser Phase stehen mehrere Beratungseinheiten zwischen der EASU und den Lehrenden im Vordergrund, die zum einen der inhaltlichen Ausgestaltung zum anderen der organisatorischen Absprache des E-Klausur dienen.

Die erste Beratungseinheit enthält im Wesentlichen die folgenden Punkte:

- Vertraut machen mit dem inhaltlichen Aufbau einer E-Klausur, den unterschiedlichen Fragetypen sowie dem Unterschied zwischen automatischer Korrektur des Systems und manueller elektronischer Korrektur.

- Erörterung der für die Durchführung einer E-Klausur relevanten prüfungsrechtlichen Grundlagen, insbesondere dort, wo die inhaltliche Ausgestaltung 
organisatorische Konsequenzen nach sich zieht. Zum Beispiel die Notwendigkeit eines Zweitprüfers oder einer Zweitprüferin, wenn eine bestimmte Grenze der Anzahl automatisch korrigierter Aufgaben überschritten wird, oder dass den Studierenden vorab die Möglichkeit gegeben werden muss, das Prüfungssystem kennenzulernen.

- Ein Überblick über die wesentlichen Schritte des E-Klausur-Prozesses, an denen die Beteiligung der Lehrenden erforderlich sein wird oder optional sein kann. So zum Beispiel bei der inhaltlichen Ausgestaltung der elektronischen Fragestellungen, während der Durchführung der E-Klausur sowie der elektronischen Korrektur.

- Danach erfolgt in der Regel die erste Entscheidung, ob die Lehrenden tatsächlich die schriftliche Klausur als E-Klausur schreiben wollen. Dem schließt sich eine Absprache der Lehrenden mit der jeweiligen Studiengangsleitung über das Vorhaben an.

Nach dieser Beratungseinheit erhalten die Lehrenden ein Dokument mit der detaillierten Beschreibung aller zur Verfügung stehenden elektronischen Fragetypen sowie eine Zusammenfassung mit den rechtlichen Anforderungen an eine E-Klausur.

Die zweite Beratungseinheit umfasst in der Regel die Festlegung, wie genau bei der Anfertigung der elektronischen Fragestellungen vorgegangen wird.

Nach Durchführung mehrerer E-Klausuren im optes-Projekt konnten wir hier zwei wesentliche Vorgehensweisen identifizieren:

\section{Variante: Technische Umsetzung der E-Fragen durch Lehrende selbst}

Diese wird von externen Lehrenden favorisiert und beinhaltet die weitestgehend selbstständige elektronische Umsetzung der E-Fragen sowie deren Zusammenfassung in der E-Klausur. Hierfür wird den Lehrenden eine detaillierte Einführung in die Umsetzung der einzelnen Fragetypen und deren Optionen sowie den Umgang mit Fragenpools und Testobjekten angeboten. Sofern ältere Papierklausuren vorliegen, erstellt die EASU einen kleinen Fragenpool mit elektronischen Umsetzungsbeispielen, an denen sich die Lehrenden bei der Erstellung ihrer neuen EFragen orientieren können. Dieses Vorgehen hat insbesondere für externe Lehrende den Vorteil, dass sie ihre E-Klausur zeitlich und räumlich unabhängig von weiteren Beratungen mit der EASU erstellen können und nur bei Bedarf Rücksprache halten.

Für die EASU hat diese Vorgehensweise allerdings den Nachteil, dass erst relativ spät eine unter Umständen sehr komplexe E-Klausur für den technischen Test durch die EASU übergeben wird. Werden hierbei Fehler in der Darstellung oder Auswertung festgestellt, steigt der Personal- und Zeitaufwand sprunghaft an, da noch kurz vor dem Klausurtermin Fehler gesucht, Korrekturen vorgenommen oder alternative Lösungen erarbeitet und mit den Lehrenden besprochen werden müssen. 


\section{Variante: Technische Umsetzung der E-Fragen durch die EASU}

Der zweite Weg wird eher von internen Lehrkräften, in der Regel hauptamtlich lehrenden Professor*innen, bevorzugt. Dabei wird auf der Grundlage vorhandener Aufgaben aus Papierklausuren erstmal ein Entwurf der elektronischen Aufgabenstellung in einem Word- oder PowerPoint-Dokument erstellt. Entsprechend dem jeweils vorgesehenen Fragetyp werden alle Texte und Antwortalternativen, deren Lösung, die vorgesehene Auswertung sowie auch mediale Elemente durch die Lehrenden vorbereitet.

Dieses Vorgehen hat den Vorteil, dass die E-Fragen in mehreren Etappen über das Semester verteilt ausgearbeitet und umgesetzt werden können. Kleine Fehler oder alternative Umsetzungsvorschläge können mit den Lehrenden schrittweise erörtert werden. Die Lehrenden haben den Vorteil, sich erst mal nicht mit der technischen Umsetzung befassen zu müssen. Allerdings liegt diese hier bei der EASU. Bei einer umfassenden Vorarbeit durch die Lehrenden kann die Aufgabe der Erstellung allerdings auch an studentische Hilfskräfte übergeben werden, mit anschließender Kontrolle durch die Mitarbeiter*innen der EASU. Dieses Vorgehen trägt dazu bei, dass personelle und zeitliche Ressourcen besser eingeteilt und zeitlich flexibler geplant werden können.

Des Weiteren werden die organisatorischen Rahmenbedingungen festgelegt. Dazu gehört die Anzahl der mit der E-Klausur zu prüfenden Studierenden und daraus resultierend die Anzahl der gleichzeitig benötigten PC-Räume und Aufsichtspersonen. Es ist weiter zu klären, in welcher Form die Studierenden mit der neuen Prüfungsumgebung vertraut gemacht werden sollen. Die meisten Lehrenden nutzen diese Auflage, indem sie den Studierenden eine vollwertige elektronische Probeklausur, circa 14 Tage vor der elektronischen Hauptklausur, mit anschließender Konsultation anbieten. Der rechtlichen Anforderung wird aber auch ein elektronischer Vortest beliebigen Inhalts gerecht, der alle Fragetypen mit ihren Optionen enthält, die auch in der Hauptklausur zum Einsatz kommen.

Es werden weiterhin organisatorische Rahmenbedingungen des jeweiligen Studiengangs in Hinblick auf die elektronische Archivierung und die Einsichtnahme durch die Studierenden geklärt. Da hier noch keine endgültige Rechtssicherheit geschaffen werden konnte, richtet die EASU sich an dieser Stelle nach den individuellen Vorgaben des Studiengangs. Ebenfalls abgestimmt wird an dieser Stelle, ob der E-Klausur-Arbeitsplatz der Studierenden noch mit zusätzlichen Dokumenten ausgestattet werden soll, zum Beispiel Notizblätter oder ein Klausurdeckblatt mit Arbeitsanweisungen und Punkteübersicht.

Im Ergebnis dieser Beratungseinheit werden von der EASU die Termin- und Raumreservierungen für die elektronische Probe- und Hauptklausur vorgenommen sowie die technischen Aufsichten von Seiten der EASU und die allgemeinen Aufsichten durch das Sekretariat des Studiengangs organisiert. Außerdem wird vom zuständigen Sekretariat bzw. den Studiengangsmanager*innen eine Liste mit den 
Matrikelnummern der zu prüfenden Studierenden übergeben, auf deren Grundlage später die Prüfungsaccounts für die Studierenden erstellt werden.

Die Lehrenden erhalten durch die EASU einen eigenen Zugang zur ILIAS-Produktivplattform und umfassende Bearbeitungsrechte für einen Arbeitsbereich, in dem sie eigene Fragenpools mit elektronischen Fragestellungen und Testobjekte erstellen bzw. als Ergebnis der Erstellung ihre Anwendung ansehen und testen können.

Es schließen sich nun in der Regel weitere Beratungseinheiten an, in denen entweder weitere technische Umsetzungsmöglichkeiten im Detail vorgestellt und besprochen werden oder fertiggestellte E-Fragen nachbearbeitet und abgenommen werden. Insbesondere werden die Lehrenden mit den Auswirkungen verschiedener optionaler Einstellungen auf die Auswertung der Aufgabenstellung vertraut gemacht. Die didaktische Ausgestaltung bei der Formulierung der Fragestellungen kann in diesen Beratungseinheiten an Bedeutung zunehmen. Die EASU bietet interessierten Lehrenden dabei Orientierung an bei der lernzielorientierten Fragenkonstruktion und deren Umsetzung in anspruchsvollen elektronischen Prüfungsfragen an.

Beabsichtigen die Lehrenden, bei der E-Klausur persönlich anwesend zu sein, wird in der direkten Vorbereitung der E-Klausur noch eine Beratung zum detaillierten Ablauf und den Aufgaben der weiteren Aufsichtspersonen während E-Klausuren durchgeführt.

Eine abschließende Beratungseinheit wird für die Einführung in die elektronische manuelle Korrektur der einzelnen Fragetypen wie Freitext- oder Zeichenaufgabe und die elektronische Nachkorrektur der Klausuraufgaben insgesamt vorgesehen. Diese Beratung kann noch vor der Durchführung der E-Klausur oder auch danach vorgenommen werden.

Die hier beschriebene Vorbereitungsphase macht den Aufwand einer Erstberatung von Seiten der EASU deutlich. Dieser reduziert sich nach der mehrmaligen Durchführung und mit fortschreitender Expertise der Lehrenden erheblich.

In der Folge beschränken sich die Beratungen auf die Einführung von Neuerungen der ILIAS-Plattform, zum Beispiel die Nutzung neuer Features oder die Verfügbarkeit von Plugins nach einem Update der Plattform. In diesem Zusammenhang wird auch über notwendige Überarbeitungen bereits vorhandener E-Fragen sowie E-Klausuren informiert.

Notwendige organisatorische Absprachen sind bekannt und können nun nach Checkliste auch kurz per Telefon oder E-Mail kommuniziert werden.

Im Ergebnis der Beratungseinheiten liegen die elektronische Probe- und die Hauptklausur fertig erstellt auf der Produktivplattform vor. Alle organisatorischen Fragen wurden besprochen und die Termin- und Raumplanung ist abgeschlossen.

Die direkte Vorbereitung der E-Klausur beginnt optimalerweise vier Wochen vor der elektronischen Hauptklausur und circa 14 Tage vor der Probeklausur. Alle weiteren Handlungsschritte werden nun von der EASU durchgeführt. 
Die nachfolgenden Schritte müssen für die Probe- und die Hauptklausur in gleicher Weise vorgenommen werden, wobei der Ablauf Probeklausur in Absprache mit den Lehrenden sinnvoll modifiziert werden kann. Handelt es sich bei der elektronischen Hauptklausur zum Beispiel um die zweite E-Klausur in einem zweisemestrigen Modul und alle Studierenden haben bereits im ersten Semester eine Probeklausur absolviert, kann ganz auf die Durchführung der Probeklausur verzichtet werden. Der rechtliche Anspruch, dass sich alle Studierenden mit dem System vertraut machen können, wurde hier bereits mit der Probeklausur im ersten Semester erfüllt.

Für die an der E-Klausur teilnehmenden Studierenden werden Prüfungsaccounts erstellt. Diese werden den Studierenden erst an ihrem jeweiligen Rechnerarbeitsplatz zur Prüfung ausgegeben. Die Weitergabe der Daten an andere Studierende ist damit ausgeschlossen. Des Weiteren wird so einer Vorgabe der DHBW Karlsruhe für schriftliche Prüfungen entsprochen. Nach dieser sollen die Lehrenden während der Korrektur die Namen der Studierenden nicht sehen, um die Gleichbehandlung der Studierenden zu gewährleisten.

Danach wird auf der der ILIAS-Prüfungsplattform ein Kursobjekt mit der Kursbezeichnung des jeweiligen Studiengangs angelegt. Dem Kursobjekt werden die vorbereiteten Prüfungsaccounts zugeordnet.

Nach abschließender Freigabe der E-Klausur auf der ILIAS-Produktivplattform durch die Lehrenden werden keine inhaltlichen Änderungen an der E-Klausur mehr vorgenommen. Die E-Klausur wird aus der Produktivplattform exportiert und auf der Prüfungsplattform in das vorbereitete Kursobjekt importiert. Es folgen die Testeinstellungen für die E-Klausur, diese werden im Vieraugenprinzip von den Mitarbeiter*innen der EASU vorgenommen. Mit den Testeinstellungen wird das technische Verhalten des Testobjekts in ILIAS festgelegt. Erst diese Einstellungen legen fest, dass es sich bei dem Test um eine E-Klausur handelt und nicht zum Beispiel um einen Selbsttest. Es werden hier beispielsweise folgende Einstellungen vorgenommen:

- dass nur ein Klausurdurchlauf pro studierender Person durchgeführt werden kann,

- dass für den Start der Klausur ein Passwort eingegeben werden muss,

- dass die Klausur sich nach Ablauf einer bestimmten Zeit automatisch schließt,

- dass die Ergebnisse nicht sofort eingesehen werden können und

- welche Elemente der Klausurumgebung den Studierenden angezeigt werden sollen.

Abschließend wird die Startseite für die Arbeitsplätze im PC-Raum eingerichtet, diese führt die Studierenden zu Beginn direkt zur E-Klausur.

Nachdem die technischen Vorbereitungen der Klausur abgeschlossen sind, werden nun noch alle notwendigen Dokumente für die Aushänge in den PC-Räu- 
men und an den Arbeitsplätzen vorbereitet und ausgedruckt. Alle für die Durchführung der E-Klausur notwendigen Materialien und Dokumente werden zusammengestellt und für den Transport in die Prüfungsräume vorbereitet.

\subsubsection{Die Durchführungsphase}

Die Phase der Durchführung umfasst die Abläufe des Prüfungstages. Sie beginnt, je nachdem wie viele PC-Räume vorzubereiten sind und wie viel technisches Personal an diesem Tag zur Verfügung steht, zwei Stunden bis eine Stunde vor Prüfungsstart. Bei der Raumreservierung ist die Vor- und Nachbereitung der PCRäume mit einzuplanen.

\section{Vor Beginn der Klausur}

Bei der Vorbereitung des jeweiligen PC-Raums werden die Sitzpläne mit den Matrikelnummern sowie Hinweisschilder auf die Klausur und die Verteilung der Studierenden auf mehrere PC-Räume gut sichtbar angebracht. Danach werden auf jedem Rechner die Prüfungsumgebung und der Safe Exam Browser mit der Startseite der E-Klausur gestartet. An jedem vorgesehenen Arbeitsplatz wird ein Sichtschutz aufgestellt (siehe Abbildung 1) und nach Sitzplan die individuellen

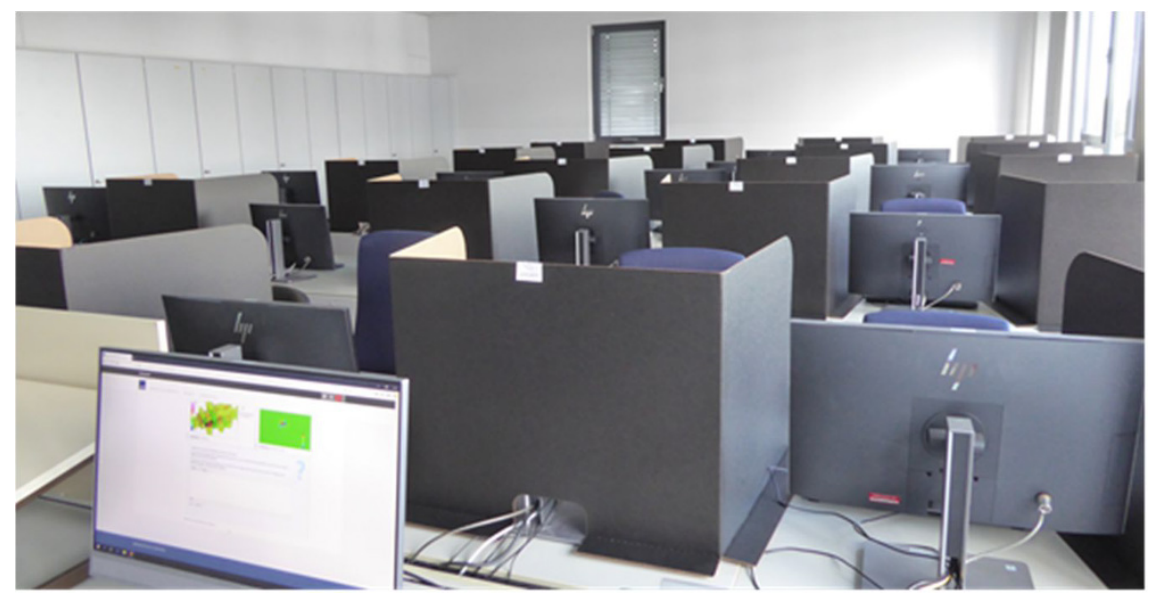

Abbildung 1: Für die E-Klausuren vorbereiteter PC-Raum an der DHBW Karlsruhe (Foto: Manfred Daniel).

Dokumente, wie ein speziell für jeden Platz vorbereitetes Beiblatt mit dem Prüfungsaccount auf der Rückseite und weitere für die Prüfung als Hilfsmittel zuge- 
lassene Dokumente ausgeteilt. Im PC-Raum werden außerdem zwei weitere Rechner als Ausweicharbeitsplatz vorbereitet, um im Falle eines Rechnerausfalls ein schnelles Umsetzen der Studierenden vornehmen zu können.

Die Studierenden orientieren sich beim Betreten des Raums am Sitzplan und nehmen den für ihre jeweilige Matrikelnummer vorgesehenen Arbeitsplatz ein (siehe Abbildung 2).

Die E-Klausur wird von zwei Aufsichten begleitet. Eine allgemeine Aufsicht übernimmt die Aufgaben, wie sie auch in Papierklausuren üblichen sind, wie die Belehrung, das Führen des Protokolls und die Kontrolle der Anwesenheit. Eine technische Aufsicht informiert die Studierenden über die Schritte zur Anmeldung in der Prüfungsumgebung und gibt deren Beginn sowie gegebenenfalls das Testpasswort zum Start der E-Klausur bekannt.

\section{Während der Klausur}

Mit der erfolgreichen Eingabe des Testpasswortes startet die E-Klausur für alle Studierenden individuell und der jeweilige Klausur-Timer zeigt kontinuierlich die Restminuten der Klausurlaufzeit an.

Die E-Klausur mit der Eingabe eines Testpasswortes zu starten, ist eine Option, die in den Testeinstellungen festgelegt werden kann. Die Nutzung dieser Option hat den Vorteil, dass man dadurch einen relativ zeitgleichen Start und damit auch einen einheitlichen Abschluss der Klausurbearbeitung erhält und so längere Wartezeiten durch erheblich zu frühe oder zu späte Abgabe einzelner E-Klausuren vermeiden kann.

Die technische Aufsicht unterstützt die Studierenden, wenn es zu technischen Schwierigkeiten während der E-Klausur kommt. Im Falle eines Rechnerversagens werden die Studierenden an einen freien Platz umgesetzt und zur letzten bearbeiteten Stelle der Klausur geführt. Die Zeit von Beginn des Ausfalls bis zur Wiederaufnahme der Bearbeitung wird den Studierenden durch elektronische Verlängerung seiner Bearbeitungszeit gutgeschrieben.

Die kontinuierlichen Fortschritte der Studierenden während der Bearbeitung ihrer E-Klausuren können durch die Aufsichten im Dashboard und der Ergebnisübersicht von ILIAS mitverfolgt und damit auch ein Gesamtbild über den reibungslosen Ablauf und den aktuellen Bearbeitungstand der E-Klausur eingeholt werden.

Während der Laufzeit der E-Klausur wird jedem und jeder Studierenden die von ILIAS generierte individuelle Prüfungsnummer eingeblendet. Die Studierenden füllen während der Klausur ein Beiblatt aus, auf dem sie ihre ILIAS-Prüfungsnummer eintragen und mit ihrer Unterschrift bestätigen, dass sie diese E-Klausur eigenständig und nur mit den zugelassenen Hilfsmitteln bearbeitet haben. Damit ist die eindeutige und von den Studierenden handschriftlich bestätigte Zuordnung einer bestimmten E-Klausur zu den jeweiligen Studierenden sichergestellt. Die 
Beiblätter werden von der technischen Aufsicht eingesammelt und für die Archivierung verwahrt.

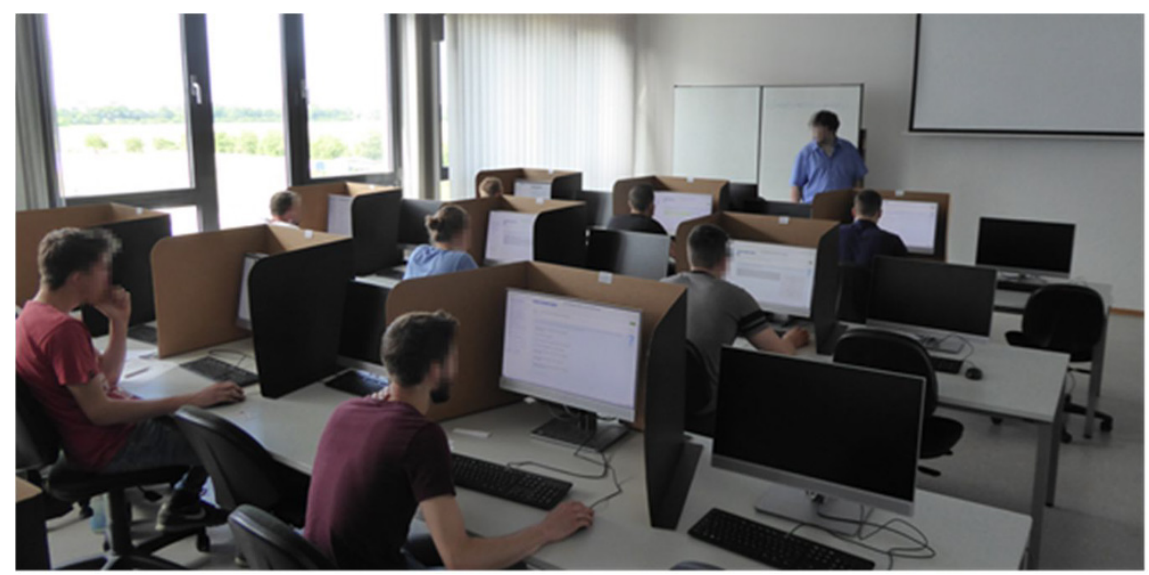

Abbildung 2: Studierende der DHBW Karlsruhe bei der Durchführung einer E-Klausur (Foto: Manfred Daniel).

Vor Ablauf der E-Klausur können sich die Studierenden eine Übersicht mit allen von ihnen gegebenen Antworten anzeigen lassen. Aus dieser Übersicht kann während der Laufzeit der E-Klausur jederzeit wieder zur Bearbeitung der Aufgabenstellungen zurückgekehrt werden (siehe Abbildung 3).

Die Abgabe der Klausur auf elektronischem Weg erfolgt entweder vor Ablauf der Klausurzeit auf Veranlassung der Studierenden oder bei Ablauf des Timers automatisch durch das System.

Nach der elektronischen Abgabe sammelt die allgemeine Aufsicht weitere Klausurdokumente wie Notiz- oder Hilfsblätter ein, sofern diese sichergestellt werden sollen.

\section{Nach Abschluss der Klausur}

Unmittelbar nach der elektronischen Abgabe aller Klausuren nimmt die technische Aufsicht die erste Archivierung sowie die Sicherung der E-Klausur mit den Teilnehmerdaten vor. Beide Dateien werden exportiert, extern aufbewahrt und gesichert.

Danach werden die PC-Räume wieder in ihren Ausgangszustand versetzt. Der Sichtschutz wird entfernt und der Safe Exam Browser sowie die Prüfungsumgebung an den Arbeitsplätzen geschlossen. Alle Prüfungsmaterialien und Dokumente werden aus den PC-Räumen abtransportiert und wieder in den Räumlichkeiten der EASU verwahrt. 


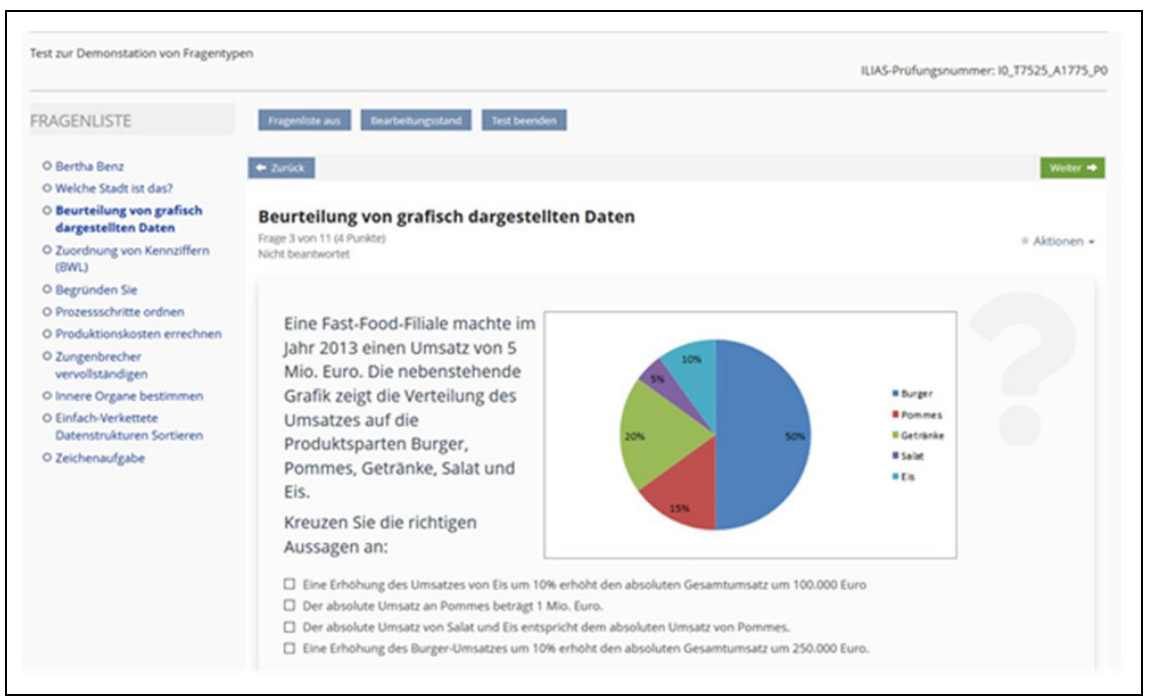

Abbildung 3: E-Klausur aus der Studierendenperspektive mit Fragenliste links und der ILIAS-Prüfungsnummer rechts oben

\subsubsection{Die Nachbereitungsphase}

Nach Beendigung der E-Klausur wird den Lehrenden für die Korrektur ein Zugang für die Prüfungsplattform eingerichtet. Die Lehrenden können die Korrektur auBerhalb der DHBW Karlsruhe nur mit einem sicheren VPN-Zugang (VPN steht für „Virtual Private Network“) ausführen.

Die Lehrenden erhalten von der EASU eine Mail mit den Zugangsdaten und dem Hinweis, dass mit der Korrektur der E-Klausur unter dem mitgelieferten Link ab sofort begonnen werden kann.

\section{Die Korrektur}

Die Lehrenden können nun zwei Arten von Korrekturmöglichkeiten ausführen. Alle E-Fragen, die nicht vom System automatisch korrigiert wurden, werden einer manuellen Korrektur unterzogen. Dies können zum Beispiel Freitextaufgaben oder Zeichenaufgaben sein, die von den Studierenden bearbeitet wurden. Die manuelle Korrektur kann in ILIAS nach Teilnehmer*in oder nach Aufgabe sortiert durchgeführt werden. Bei der Korrektur nach Teilnehmer*in wird eine Klausur nach der anderen fertig korrigiert. Bei der Korrektur nach Aufgabe wird immer erst eine bestimmte Aufgabenstellung für alle Teilnehmer*innen korrigiert, danach die nächste Aufgabe und so weiter. 
Neben der manuellen Korrektur ist die Nachkorrektur der E-Klausur möglich. Diese kommt zum Beispiel zum Einsatz, wenn ein Fehler in einer Aufgabe entdeckt wird und diese Aufgabe für alle Teilnehmer*innen aus der Klausur entfernt werden soll oder eine häufige Antwort im Vorfeld nicht als Lösung hinterlegt wurde, rückwirkend aber für alle Teilnehmer*innen als richtig gewertet werden soll.

\section{Die Einsichtnahme}

Nachdem die Lehrenden der EASU den Abschluss ihrer Korrektur mitgeteilt haben, kann die Einsichtnahme der Studierenden vorbereitet werden. Die Einsichtnahme von E-Klausuren ist in den Studiengängen der DHBW Karlsruhe noch unterschiedlich geregelt. In manchen Studiengängen ist eine Einzeleinsicht und darüber hinaus mit dem Papierausdruck der E-Klausur vorgesehen.

Für die elektronische Einsichtnahme wird mit Beginn der Lehrveranstaltungen des neuen Semesters ein Einsichtstermin in den PC-Räumen der DHBW Karlsruhe organisiert, der gut in den Studienplan der Studierenden passt.

Die korrigierten Klausurergebnisse werden durch die EASU zur Einsichtnahme für die Studierenden freigegeben. Die Studierenden erscheinen kursweise oder nacheinander zum festgelegten Termin und sehen sich ihre Ergebnisse in einer Gegenüberstellung ihrer Lösung mit Korrekturhinweisen und der bestmöglichen Lösung an.

Haben die Studierenden Grund zur Beanstandung, können Beanstandungsformulare ausgefüllt werden, die im Anschluss an die Lehrenden weitergeleitet werden. Die Lehrenden beantworten jede Beanstandung mit deren Anerkennung oder Ablehnung, wie es auch in Papierklausuren üblich ist.

\section{Die Archivierung}

Auch die Archivierung der elektronischen Klausuren ist an der DHBW Karlsruhe noch nicht abschließend einheitlich festgelegt. Die übliche elektronische Vorgehensweise ist die Erstellung einer Archivierungsdatei als ZIP-Archiv in ILIAS sowie deren Export und Speicherung auf Speichermedien, die eine sichere Aufbewahrung der E-Klausur-Archivdatei über die vorgeschriebene Aufbewahrungszeit ermöglichen. Die Sekretariate bevorzugen aufgrund ihrer technischen Ausstattung derzeit noch die Speicherung auf CD-ROM. Die Speicherung der Archivdateien erfolgt pro Kurs und Klausur auf einer CD-ROM, die im Klausurordner mit den Beiblättern und weiteren Klausurdokumenten verwahrt werden.

\subsection{Drei Umsetzungsbeispiele}

Im Folgenden sollen drei Umsetzungsbeispiele von E-Klausuren aus der aktuellen Lehrpraxis an der DHBW Karlsruhe vorgestellt werden. In den Beispielen werden 
die Komplexität der dargestellten Inhalte, die organisatorischen Rahmenbedingungen und die Motivation der Lehrenden, eine E-Klausur durchzuführen, geschildert. Die Beispiele sollen insbesondere die Vielfalt von Einflussfaktoren deutlich machen, welche den Arbeitsaufwand der EASU bei der Durchführung von E-Klausuren bestimmen.

\subsubsection{E-Klausur ,Simulationstechnik“ im Studiengang Maschinenbau}

Die Lehrveranstaltung Simulationstechnik im Studiengang Maschinenbau ist ein über zwei Semester durchgeführtes Modul, an dem Bachelorstudierende im dritten Studienjahr teilnehmen. Die E-Klausuren finden jeweils am Ende des 5. und 6. Semesters statt. Die Lehrveranstaltung wird von einer externen Lehrbeauftragten durchgeführt, die ihr umfassendes Spezialwissen aus ihrer Berufspraxis bei der Vermittlung der Wissensinhalte einbringt. Entsprechend komplex gestaltete sich auch die Umsetzung der E-Klausur-Inhalte in ILIAS. Es kamen vielfältige, insbesondere auch neue Fragetypen wie die Zeichenaufgabe mit ihren Optionen zum Einsatz (siehe Abbildung 4). Die Möglichkeit, komplexe Formeln mit LaTeX darzustellen, wurde nicht nur im Fragetext, sondern auch in den Antwortalternativen intensiv genutzt (siehe Abbildung 5).

Im Studienfach Simulationstechnik wird nun bereits die dritte E-Klausur geschrieben und es lässt sich an diesem Beispiel sehr gut beobachten, wie der anfänglich sehr hohe Beratungs- und Umsetzungsaufwand von Seiten der EASU mit jeder neuen E-Klausur abnimmt. Die Lehrende beherrscht nun die Erstellung von Tests und elektronischen Fragestellungen in ILIAS und wird zunehmend vertrauter mit den Einstellungen in den Optionen der Fragetypen sowie deren Auswirkungen auf die anschließende elektronische Auswertung.

Die Beratungsaufgaben der EASU beschränken sich nun in der Erstellungsphase der E-Klausur auf die Beantwortung einzelner Anfragen und die abschließende Überprüfung und den Test der Lauffähigkeit der fertigen E-Klausur unter Prüfungsbedingungen sowie die Einrichtung der E-Klausur auf der Prüfungsplattform.

Im Gegensatz dazu stieg zeitweise der Aufwand bei der Organisation dieser E-Klausur stark an, da sich die Anzahl der zum gleichen Termin zu prüfenden Studierenden von einem auf zwei Kurse und damit auf 65 Studierende erhöht hatte. Diese Anzahl machte die personelle und technische Organisation und Ausstattung von drei PC-Räumen gleichzeitig notwendig. Neben der elektronischen Hauptklausur wurde im ersten Semester der Lehrveranstaltung auch eine Probeklausur mit dem gleichen Umfang der Hauptklausur organisiert. 
Im nachfolgenden Bild ist die Geschwindigkeitskomponente $v$ in $y$-Richtung bei der Strömung um einen Zimtstern im Wind dargestellt. Aus dieser Darstellung lasst sich auf Wirbelvorkommen in der Strömung schließen.

Zeichnen Sie bitte mindestens zwei Wirbel und ihre Drehrichtung mit Hilfe von Stromlinien ein! Hinweis:

- Oberlegen Sie, wo das Wirbelzentrum sein könnte und welche Geschwindigkeit dort vorherrscht. Zeichnen

Sie die Wirbel entsprechend um das Zentrum herum.

- Hinsichtlich der Geschwindikeit im Wirbelzentrum verhalt sich ein kleiner Wirbel nicht anders als ein atmosphărischer Wirbelsturm, z.B. ein Hurrkan.

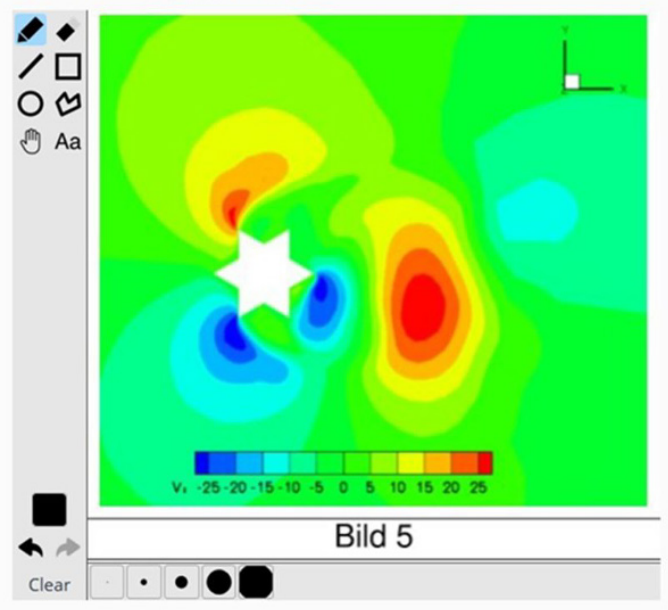

Abbildung 4: Beispiel einer E-Frage zur Strömungsanalyse unter Verwendung des Fragetyps Zeichenaufgabe

Es sollen ein explizites und ein implizites Zeitschrittverfahren für eine allgemeine, zeitabhängige Strömungsvariable $\varphi(t)$ verwendet werden.

Ordnen Sie zu, welches ein explizites und welches ein implizites Zeitschrittverfahren ist. Beachten Sie dabei, dass sich die Darstellung auf den Zeitpunkt $t_{n}$ auf der unten dargestellten Zeitachse bezieht.

Treffen Sie die Zuordnung, indem Sie die dargestellten Zeitschrittverfahren rechts in die entsprechenden, zugehörigen Definitionen links ziehen.

\begin{tabular}{|c|}
\hline$\varphi_{n+1}=\mathcal{G}\left(\varphi_{n+2}(\mathrm{x}), \varphi_{\mathrm{n}}(\mathrm{x}), \varphi_{\mathrm{n}-2}(\mathrm{x})\right)+\Delta \mathrm{t} \mathcal{F}\left(\mathrm{p}_{\mathrm{n}}\left(\mathrm{x}_{1}, \mathrm{x}_{2}\right), \mathrm{u}_{\mathrm{n}}\left(\mathrm{x}_{1}, \mathrm{x}_{2}\right)\right)$ \\
\hline$\varphi_{n+1}=\mathcal{G}\left(\varphi_{n}(\mathrm{x}), \varphi_{\mathrm{n}-1}(\mathrm{x})\right)+\Delta \mathrm{t} \mathcal{F}\left(\mathrm{p}_{\mathrm{n}}\left(\mathrm{x}_{1}, \mathrm{x}_{2}\right), \mathrm{u}_{\mathrm{n}}\left(\mathrm{x}_{1}, \mathrm{x}_{2}\right)\right)$ \\
\hline$\varphi_{n+1}=\mathcal{F}\left(\varphi_{n+1}(\mathrm{x}), \varphi_{\mathrm{n}}(\mathrm{x}), \varphi_{\mathrm{n}-1}(\mathrm{x})\right)+\Delta \mathrm{t} \mathcal{G}\left(\mathrm{p}_{\mathrm{n}}\left(\mathrm{x}_{1}, \mathrm{x}_{2}\right), \mathrm{u}_{\mathrm{n}}\left(\mathrm{x}_{1}, \mathrm{x}_{2}\right)\right)$ \\
\hline$\varphi_{n+1}=\mathcal{F}\left(\varphi_{n}(\mathrm{x}), \varphi_{\mathrm{n}-1}(\mathrm{x}), \varphi_{\mathrm{n}-2}(\mathrm{x})\right)+\Delta \mathrm{t} \mathcal{G}\left(\varphi_{\mathrm{n}}(\mathrm{x}), \mathrm{p}_{\mathrm{n}}(\mathrm{x}), \mathrm{u}_{\mathrm{n}}(\mathrm{x})\right)$ \\
\hline
\end{tabular}

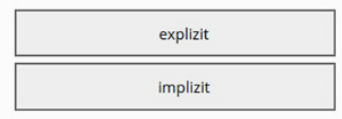

Abbildung 5: Darstellung von komplexen Formeln mit LaTeX in einer ILIAS-Zuordnungsaufgabe 
Nach Einschätzung der Lehrenden liegt der Vorteil, im Fach Simulationstechnik eine E-Klausur durchzuführen, in den besseren Darstellungsmöglichkeiten von Simulationsprozessen und auch der Lesbarkeit sowie den Korrekturmöglichkeiten in elektronischen Freitextaufgaben. Letztere und die automatische Korrektur führten nach ihren Angaben zu einer übersichtlicheren und zeitsparenden Korrektur der E-Klausur, insbesondere nach Anstieg der Studierendenzahl in ihrem Studienfach.

Von Seiten der EASU wird der bisherige Gesamtaufwand bei der Umsetzung und Organisation sowie auch die Pflege der bereits erstellten Fragenpools dieser E-Klausur eher als hoch bewertet.

\subsubsection{E-Klausur „Analysis und Lineare Algebra“ im Studiengang Wirtschafts- informatik}

Die E-Klausur Analysis und Lineare Algebra im Studiengang Wirtschaftsinformatik ist ebenfalls die erste Klausur in einem zweisemestrigen Mathematik-Modul, im Grundlagenstudium des Bachelorstudienganges. Sie bildet den Abschluss einer Lehrveranstaltung nach dem Inverted-Classroom-Modell, die ebenfalls im Projekt optes auf technischer Basis der ILIAS-Plattform pilotiert wurde. Näher beschrieben wird diese Lehrveranstaltung im Kapitel 21. Da elektronische Selbsttests und die dazugehörigen Fragenpools hier bereits ein zentraler Bestandteil der Lehrveranstaltung waren, wurde mit einer elektronischen Klausur der E-Learning-Charakter der Lehrveranstaltung bis zum Abschluss einheitlich fortgeführt. Auch hier wurde mit den Studierenden vorab eine elektronische Probeklausur durchgeführt, die den Studierenden das Kennenlernen der elektronischen Klausurbedingungen in Verbindung mit einer anschließenden Konsultation ermöglichte.

Der Dozent war als optes-Projektmitarbeiter, der die Umsetzung der Lehrveranstaltung vornahm, bereits mit der Erstellung von Tests und Fragenpools vertraut. Dem zufolge bestanden die Aufgaben der EASU in der Überprüfung der Lauffähigkeit und der Einrichtung der E-Klausur auf der Prüfungsplattform sowie der organisatorischen Umsetzung der elektronischen Probe- und Hauptklausur für 32 Studierende in zwei PC-Räumen.

Obwohl sowohl die elektronische Probe- als auch die Hauptklausur technisch und organisatorisch einwandfrei durchgeführt wurden, ist deren inhaltliche Ausgestaltung kritisch zu betrachten. Dies ist im konkreten Fall von zwei Rahmenbedingungen abhängig.

a) Im Studiengang Wirtschaftsinformatik einigen sich fünf Mathematikdozent*innen auf eine hundertprozentig einheitliche Klausur. Da in vier Kursen eine Papierklausur geschrieben wird, schränken die inhaltlichen Gestaltungsvorgaben in den Aufgabenstellungen die mögliche Nutzung vielfältiger elektronischer Fragetypen stark ein. 
b) Ein weiterer kritischer Punkt ist, dass mathematische Rechenwege und Beweise mit den Fragetypen in ILIAS nicht abgebildet werden können. Deshalb wird bei der Durchführung der E-Klausur auf eine Mischform aus elektronischer Beantwortung der Fragen und Darstellung der Rechenwege auf Papier zurückgegriffen. Während die reine Ergebnisprüfung vom ILIAS-System automatisch vorgenommen werden kann, wird zumindest bei falschen Ergebnissen der Rechenweg auf Notizpapier mit korrigiert und entsprechende Teilpunkte vergeben.

Diese beiden Bedingungen stellen den Sinn der Durchführung einer E-Klausur im Verhältnis zum organisatorischen Aufwand in Frage.

Nach Einschätzung der EASU liegt der Gesamtaufwand dieser E-Klausur im mittleren Bereich. Die Archivierung des elektronischen Teils der Klausur erfolgt auf digitalen Datenträgern gemeinsam mit den weiteren Dokumenten in Papierform. Auch die Einsichtnahme durch die Studierenden findet auf elektronischem Wege statt. Die Studierenden können ihre Klausurergebnisse zu Beginn des nächsten Semesters in den PC-Räumen einsehen.

\subsubsection{E-Klausur „Produktmanagement“ im Studiengang Wirtschaftsingenieurwesen}

Die E-Klausur Produktmanagement im Studiengang Wirtschaftsingenieurwesen ist ebenfalls Bestandteil eines zweisemestrigen Moduls „Marketing II“ im Grundlagenstudium des Bachelorstudienganges. In diesem Fall wird das Modul jedoch von einem anderen Dozenten ohne E-Klausur fortgesetzt. Die Lehrkraft des Studienfaches „Produktmanagement“ ist Professor an der DHBW Karlsruhe und neben seiner Lehrtätigkeit mit den Aufgaben eines Studiengangleiters betraut.

Aufgrund seiner vielfältigen Aufgaben ist er vor allem an den zeitökonomischen Vorteilen der Durchführung von E-Klausuren interessiert, ohne dabei Abstriche im didaktischen Anspruch der Aufgabenstellungen machen zu wollen. Obwohl sich die Inhalte der Fragestellungen sehr gut für eine elektronische Umsetzung eignen, schätzt der Lehrende den Anfangsaufwand für didaktisch anspruchsvolle elektronische Aufgabenstellungen relativ hoch ein, wie zum Beispiel bei der Formulierung von eindeutigen Antwortalternativen im Fragetyp K-Prim.

Er kann sich aber gut vorstellen, im weiteren Verlauf über mehrere Semester beim Einsatz von E-Klausuren sehr stark von der Zeiteinsparung zu profitieren und damit mehr Zeit für andere wichtige Aufgaben in seinem Studiengang zu gewinnen. Eine Zeiteinsparung sieht er hier vor allem in zwei Phasen:

- in der Erstellungsphase der E-Klausur durch Rückgriff auf eine große Sammlung von E-Fragen in einem stetig wachsenden Fragenpool und 
- in der Korrekturphase durch die Nutzung einer Vielzahl automatisch korrigierbarer Fragetypen und der verbesserten Lesbarkeit bei elektronischen Freitextaufgaben.

Mit der E-Klausur in Produktmanagement konnte der Lehrende erste wichtige Erfahrungen mit der Erstellung und Durchführung von E-Klausuren sowie deren Unterstützung durch die EASU sammeln. So wurde zum Beispiel festgestellt, dass der Fragenumfang bei einer ausschließlich automatisch korrigierten Klausur noch etwas erhöht werden muss. Alle Studierenden waren früher mit der Lösung der Aufgaben fertig und benötigten nicht den gesamten vorgesehenen Klausurzeitraum.

Obwohl die technische Erstellung der E-Fragen in diesem Fall noch bei der EASU lag, war der Gesamtaufwand der EASU bei dieser E-Klausur am geringsten. Die Erstellungs- und Vorbereitungsphase der E-Klausur verlief in mehreren Phasen über das Semester verteilt, sodass die einzelnen Arbeitsschritte gut geplant und verteilt werden konnten. Der Lehrende übergab in Etappen vollständige Entwürfe seiner E-Fragen die von der EASU schrittweise in den technischen Rahmen von ILIAS übertragen wurden. Bei der Übertragung waren keine größeren technischen Herausforderungen zu bewältigen, sodass diese auch unter Aufsicht eines Projektmitarbeiters oder einer Projektmitarbeiterin von studentischen Hilfskräften übernommen werden könnten. Es war ausreichend Zeit für Rückfragen zu Einstellungen und Auswertung der einzelnen E-Fragen zwischen der EASU und dem Lehrenden vorhanden.

Für das Kennenlernen der Prüfungsumgebung durch die Studierenden musste keine zusätzliche Probeklausur erstellt werden. Es wurde ein Test mit beliebigen Inhalten genutzt, der alle Fragetypen enthielt, welche auch in der elektronischen Hauptklausur zum Einsatz kamen.

Die E-Klausur wurde für insgesamt 22 Studierende durchgeführt. Dies reduzierte den organisatorischen Aufwand, da hier noch der größte einzelne PC-Raum genutzt werden konnte.

\subsubsection{Erkenntnisse aus den Beispielen}

Unabhängig vom Erstaufwand und den damit verbundenen Beratungsleistungen durch die EASU lassen sich bei der Analyse dieser Beispiele folgende Gruppen von Einflussfaktoren identifizieren, die den Aufwand der EASU bei der Durchführung einer E-Klausur bestimmen:

- Die Komplexität der umzusetzenden Inhalte und damit, wie herausfordernd sich die Umsetzung der E-Fragen gestaltet.

- Das Ausmaß der Arbeitsbereiche und Aufgaben der EASU, an denen auch studentische und wissenschaftliche Hilfskräfte beteiligt werden können. 
- Die Rolle der Lehrenden, für die die E-Klausur ausgeführt werden soll: externe nebenberufliche oder interne hauptberufliche Lehrkraft.

- Die Anzahl der gleichzeitig elektronisch zu prüfenden Studierenden bei begrenzten räumlichen Ressourcen.

- Die organisatorischen Rahmenbedingungen des Studiengangs und deren Auswirkung auf die Workflows im E-Klausur-Prozess.

\subsection{Rahmenbedingungen für eine weitere Verstetigung}

Da für die Durchführung von Lehrveranstaltungen an der DHBW Karlsruhe derzeit die Lernplattform Moodle genutzt wird, gibt es zwei mögliche Vorgehensweisen bei der technischen Verstetigung:

1) Die technische Verbindung beider Lernplattformen Moodle und ILIAS im Bereich des E-Assessment, zum Beispiel durch die Nutzung einer LearningTools-Interoperability (LTI)-Schnittstelle oder

2) die Übertragung des E-Klausur-Prozesses auf die Lernplattform Moodle, wobei bereits abzusehen ist, dass für die vollständige Durchführung von E-Klausuren ergänzende technische Verfahren erprobt werden müssen.

Die weitere Übernahme der E-Klausur-Unterstützung durch das Education Support Center (ESC) der DHBW Karlsruhe ist abhängig vom möglichen Einsatz weiterer personeller Ressourcen, die nach der Beendigung des Projekts optes diese Aufgaben übernehmen können.

Aus ökonomischer Sicht ist die Erweiterung personeller Ressourcen jedoch nur sinnvoll, wenn der Gesamtnutzen des Einsatzes von E-Klausuren höher eingeschätzt werden kann als die dafür aufgewendeten neuen Personalressourcen. Der Gesamtnutzen ist jedoch nicht einfach und eindeutig zu bestimmen, da dieser von einer Vielzahl variabler Einzelfaktoren beeinflusst wird, die sich teilweise auch in didaktischem und gestalterischem Mehrwert ausdrücken. Kosten-Nutzen Modellrechnungen, die sich auf rein zeitökonomische Aspekte beziehen, wurden von der EASU durchgeführt. 
Der Transfer des E-Klausurprozesses als Vorgehensmodell in seiner Gesamtheit oder in Teilen ist besonders für Hochschuleinrichtungen mit ähnlichen Voraussetzungen, wie kleine Studierendengruppen zwischen 25 und 30 Studierenden und der typischen Ausstattung mit mehreren einzelnen PC-Räumen mit jeweils 30 bis 40 Arbeitsplätzen, möglich. Das Interesse weiterer Studien-akademien der DHBW an dem beschriebenen Prozess nimmt beständig zu. Seit Mitte der ersten Projektphase wird der E-Klausuren-Prozess bei unseren Projektpartnern an der Technischen Hochschule Ostwestfalen-Lippe erfolgreich angewendet sowie an die Rahmenbedingungen der eigenen Einrichtung angepasst und erweitert.

Open Access Dieses Kapitel wird unter der Creative Commons Namensnennung 4.0 International Lizenz (http://creativecommons.org/licenses/by/4.0/deed. de) veröffentlicht, welche die Nutzung, Vervielfältigung, Bearbeitung, Verbreitung und Wiedergabe in jeglichem Medium und Format erlaubt, sofern Sie den/ die ursprünglichen Autor(en) und die Quelle ordnungsgemäß nennen, einen Link zur Creative Commons Lizenz beifügen und angeben, ob Änderungen vorgenommen wurden.

Die in diesem Kapitel enthaltenen Bilder und sonstiges Drittmaterial unterliegen ebenfalls der genannten Creative Commons Lizenz, sofern sich aus der Abbildungslegende nichts anderes ergibt. Sofern das betreffende Material nicht unter der genannten Creative Commons Lizenz steht und die betreffende Handlung nicht nach gesetzlichen Vorschriften erlaubt ist, ist für die oben aufgeführten Weiterverwendungen des Materials die Einwilligung des jeweiligen Rechteinhabers einzuholen.

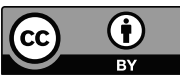

